\title{
POISSON REDUCTION OF THE SPACE OF POLYGONS
}

\author{
IAN MARSHALL
}

\begin{abstract}
A family of Poisson structures, parametrised by an arbitrary odd periodic function $\phi$, is defined on the space $\mathcal{W}$ of twisted polygons in $\mathbb{R}^{\nu}$. Poisson reductions with respect to two Poisson group actions on $\mathcal{W}$ are described. The $\nu=2$ and $\nu=3$ cases are discussed in detail and the general $\nu$ case in less detail. Amongst the Poisson structures arising in examples are to be found the lattice Virasoro structure, the second Toda lattice structure and some extended Toda lattice structures. A general result is proved showing that, for any $\nu$, to certain concrete choices of $\phi$ there correspond compatible Poisson structures which generate all the extended bigraded Toda hierarchies of a suitable size.
\end{abstract}

\section{INTRODUCTION}

The space $\mathcal{D}_{\nu}$ of scalar differential operators $L$ of order $\nu$ with periodic coefficients, (1) $\mathcal{D}_{\nu} \ni L=\partial^{\nu}+u_{\nu-1} \partial^{\nu-1}+\cdots+u_{1} \partial+u_{0}, \quad \partial=d / d x, \quad u_{k} \in C^{\infty}(\mathbb{R} / 2 \pi \mathbb{Z}, \mathbb{R})$,

is a Poisson subspace, with respect to two compatible Poisson structures, of the space of periodic pseudo-differential operators. These Poisson structures are usually associated with the names of Adler and of Gelfand-Dikii, see [A, GD. Alternatively $\mathcal{D}_{\nu}$ may be subjected to analysis via a Hamiltonian reduction procedure [DS], known in this context as Drinfeld-Sokolov reduction, which results in the same pair of Poisson structures. The reduction procedure rests on the observation that in writing the equation $L \psi=\psi$ as a $\nu \times \nu$ matrix system $\partial \Psi+\mathcal{L} \Psi=0$, the matrix $\mathcal{L}$ is not uniquely defined. Let $\mathfrak{b}=$ \{lower triangular matrices $\}$ and $\mathfrak{n}:=$ \{strictly lower triangular matrices $\}. \mathfrak{b}$ and $\mathfrak{n}$ are Lie subalgebras of $g l_{\nu}$ with associated Lie groups $B=\{$ lower triangular matrices $\} \cap G L_{\nu}$ and $N=\{A+I d \mid A \in \mathfrak{n}\}$. Defining the matrix $\Lambda=\sum_{i=1}^{\nu-1} E_{i, i+1}$

$$
\Lambda=\left(\begin{array}{cccc}
0 & 1 & \cdots & 0 \\
\vdots & \ddots & \ddots & \vdots \\
\vdots & \cdots & \ddots & 1 \\
0 & \cdots & \cdots & 0
\end{array}\right),
$$

then $A \in \mathfrak{b}$ may be chosen so that $\mathcal{L}=-\Lambda-A$. A canonical choice for $A$ is

$$
A_{\text {can }}=\left(\begin{array}{ccc}
0 & \cdots & 0 \\
\vdots & & \vdots \\
0 & \cdots & 0 \\
u_{0} & \cdots & u_{\nu-1} \\
1 & 1
\end{array}\right),
$$


corresponding to $\Psi_{\text {can }}^{T}=\left(\psi, \psi^{\prime}, \cdots, \psi^{(\nu-1)}\right)$. An equivalent representation of $L \psi=0$ can be obtained by making a different choice for $\Psi$. Thus $\Psi$ may be replaced by $\hat{\Psi}=g \Psi_{\text {can }}$ for some $g \in N$ whose entries are periodic functions, to get $\partial \hat{\Psi}+\hat{\mathcal{L}} \hat{\Psi}=0$. In other words, we have a gauge freedom in the passage from $L$ to $\mathcal{L}$ via the action of the nilpotent subgroup $N$ of strictly lower triangular matrices: $\mathcal{L} \sim \hat{\mathcal{L}} \Leftrightarrow \exists g \in N$ s.t. $\hat{\mathcal{L}}=g \mathcal{L} g^{-1}+(\partial g) g^{-1}$. That is $\mathcal{D}_{\nu}=\{\mathcal{L}=-\Lambda+A \mid A \in \mathfrak{b}\} / N$. The key to the Drinfeld-Sokolov reduction procedure is the identification of a Poisson structure on the space of $\mathcal{L}_{\mathrm{s}}$ with respect to which the gauge action is Poisson. In fact this is the standard Lie-Poisson structure on the dual of the central extension of the loop algebra of $g l_{\nu}$. Fixing the form of $\mathcal{L}$ to be $\mathcal{L}=-\Lambda+A$ with $A$ a lower-triangular matrix is the fixing of the momentum map for the gauge action. The construction may be extended to an arbitrary semi-simple Lie group $G$, replacing the role of $\Lambda$ by a principal nilpotent element in $\mathfrak{g}=\operatorname{Lie}(G)$, and by replacing the group of lower triangular matrices by the appropriate Borel subgroup and the strictly lower ones by the appropriate nilpotent subgroup.

An analogous construction was undertaken in the articles [FRS] and [SS] for $q$ difference operators and for shift operators on a one-dimensional lattice. From now on everything will be in the setting of the one-dimensional lattice, although most, if not all, of what follows here applies equally to the $q$-difference case 11 One would like to discover a Poisson structure on the space $\mathcal{S}_{\nu}$ of scalar shift-operators $L$ of order $\nu$ with periodic coefficients,

$$
\begin{aligned}
\mathcal{S}_{\nu} \ni L=D^{\nu}+u_{\nu-1} D^{\nu-1}+\cdots+u_{1} D+u_{0}, & \\
(D f)_{m}=f_{m+1} \text { for } f \in F u n(\mathbb{Z}, \mathbb{R}) & =\text { infinite sequences in } \mathbb{R}, \\
u_{k} \in F u n(\mathbb{Z} / N \mathbb{Z}, \mathbb{R}) & =\text { periodic sequences in } \mathbb{R} .
\end{aligned}
$$

Exactly the same argument as described above for the operators $L \in \mathcal{D}_{\nu}$ in (11) holds for the operators $L \in \mathcal{S}_{\nu}$ in (2), except that now the freedom in the choice of representation of the scalar equation is generated by $\hat{\Psi}=g \Psi$ for $g \in N$ with entries in $F u n(\mathbb{Z} / N \mathbb{Z}, \mathbb{R})$. One arrives at the point where to cast the reduction procedure in a Poisson setting, a Poisson structure is required on the space of $\mathcal{L}$ s, with respect to which the gauge-action $\hat{\mathcal{L}}=(D g) \mathcal{L} g^{-1}$ be Poisson: this is provided by the one due to Semenov-Tian-Shansky, given in [S1] the label twisted lattice current algebra. Hence the natural setting for the Poisson description of difference operators is that of Poisson Lie groups and it involves the choice of a suitable $r$-matrix. The construction may also be extended to the cases for which $G L_{\nu}$ is replaced by arbitrary semisimple Lie groups.

The Poisson algebras resulting from the Drinfeld-Sokolov setting are known, in the language of Conformal Field Theory, as W-algebras. Those resulting from the Frenkel-Reshetikhin-SemenovTianShansky-Sevostyanov setting are known by analogy as $q$-deformed or lattice $\mathrm{W}$-algebras. The simplest example of a $\mathrm{W}$-algebra is the Virasoro algebra, which comes from the $s l_{2}$ case of reduction. The corresponding

\footnotetext{
${ }^{1}$ It would be of some interest - at least to this author - to find the $q$-difference version of (6).
} 
lattice W-algebra is called, by analogy, the lattice Virasoro algebra. An equivalent lattice analogue of Virasoro is the Faddeev-Takhtajan-Volkov algebra. As was described in [FRS there is a map between the two algebras. This is discussed in Section 2 of the present article.

In both the Drinfeld-Sokolov setting for differential operators and the FrenkelReshetikhin-SemenovTianShansky-Sevostyanov setting it is perceived - either as a useful observation in the DS setting, or as a crucial requirement in the FRS-SS setting - that fixing the form of $\mathcal{L}$ to $\mathcal{L}=\Lambda+A$, with $A$ lower-triangular, is a constraint of first class type. Indeed this was invoked in [FRS] and in [SS] as a device by means of which the exact form of the $r$-matrix they needed was to be fixed uniquely.

In the present article there will be presented an alternative way to obtain a Poisson structure on the space $\mathcal{S}_{\nu}$ of shift operators. The computations will be presented in fullest detail only for second and third order examples, but this is enough to illustrate the principal idea which, as in the articles [FRS, SS], is to first identify the space $\mathcal{S}_{\nu}$ as a quotient space of a more elementary space, and then to interpret the projection from the elementary space to the quotient as a reduction using a standard argument from the theory of Poisson groups. The most striking thing is the indication that the requirement in [FRS] and [SS] for constraints to be firstclass was unnecessarily strong, and that by relaxing it the results which may be incorporated in the reduction procedure are extended.

To justify this last claim, consider the space of second order difference operators on a periodic lattice of length $N$,

$$
L=D^{2}-u D+\rho, \quad u, \rho \in F u n(\mathbb{Z} / N \mathbb{Z}, \mathbb{R}), \quad(D f)_{n}=f_{n+1} .
$$

On the one hand the space of such operators is familiar as being the same as the set of periodic tri-diagonal matrices, providing the standard setting for the Toda lattice system. On the other hand, after fixing $\rho \equiv 1$ it is a discrete analogue of the space of periodic Schrödinger operators, on which the standard Poisson structure as found in [FRS] - is identified with the Faddeev-Takhtajan-Volkov [FT, V] Poisson structure; itself a discrete analogue of the Virasoro algebra, which is in turn naturally related to standard periodic Schrödinger operators, $L=\partial^{2}+u$.

The point of view described in the present article admits an element of freedom absent in [FRS], allowing the recovery not only of the lattice Virasoro structure upon constraining $\rho \equiv 1$ in (3), but also of the "second Toda lattice Poisson bracket" without fixing $\rho$. One begins with the space $\mathcal{W}$ of quasi-periodic sequences in $\mathbb{R}^{2}$ on which a family of Poisson structures is defined, parametrised by an arbitrary odd function $\phi$ and on which the action of $G L_{2}$ is a Poisson action. The space of operators of the form given in (3) is the same as $\mathcal{W} / G L_{2}$. The function $\phi$ may be viewed as a remnant of the initial freedom (subsequently relinquished) in the definition of the $r$-matrix in [FRS, SS]. For one choice for $\phi$, which corresponds to the fixing of the $r$-matrix imposed in [FRS, [SS], $\rho \equiv 1$ is a first-class constraint and one obtains the lattice Virasoro algebra directly. For another choice, which produces the Toda Poisson structure, $\rho \equiv 1$ is not a constraint of first-class type, 
IAN MARSHALL

but reduction by the Dirac method can be made nonetheless. A simple corollary, which follows from Proposition 1.6, is that constraining of the second Toda Poisson bracket - by the standard Dirac method - yields the lattice Virasoro structure. This fact appears not to have been previously published, although it was not unknown to experts $[\mathrm{D}]$. Here it is given an explanation of sorts.

It is appropriate to make the remark here that the constraint on $\rho$ may be more general; that is, for any fixed periodic $\beta$, by imposing $\rho \equiv \beta$. The result is a generalisation of the lattice Virasoro algebra in which $\beta$ furnishes a set of parameters. This more general Poisson structure appeared - apparently for the first time - in the article [VS].

The results presented in this article are closely related to those of the article [MS]. The main idea is to start with the exchange algebra defined in (6) and in Proposition 1.2. This was also the point of departure in the article [B] of Babelon which treated similar questions to the ones dealt with here.

At various stages this work was done during a number of visits to different universities and research institutes, whilst I was wandering around with no job. I wish to express my gratitude to all of my hosts, sometimes for their hospitality and encouragement, sometimes for their help and suggestions and in many cases for all of these.

\section{Poisson structure on the Space of POLygons in $\mathbb{R}^{\nu}$}

Let $N \in \mathbb{N}$ be fixed. We consider the space of twisted polygons of length $N$ in $\mathbb{R}^{\nu}$, which will be denoted $\mathcal{W}$. (It is assumed that $N$ is reasonably large compared with $\nu$. If not, then some of the arguments which follow later do not make sense.)

Note 1.1. In this article, elements in $\mathbb{R}^{\nu}$ are always written as row-vectors, except within determinants, where they will appear as column vectors.

An element of $\mathcal{W}$ is a pair $(V, M)$, where $V: \mathbb{Z} \rightarrow \mathbb{R}^{\nu} \backslash\{\mathbf{0}\}$ is a sequence in $\mathbb{R}^{\nu} \backslash\{\mathbf{0}\}$ and $M$ is an element in $G L_{\nu} . V$ and $M$ are related by the condition of quasi-periodicity or twisting, $V_{n+N}=V_{n} M \quad \forall n$. Thus we define

$$
\mathcal{W}=\left\{(V, M) \in F u n\left(\mathbb{Z}, \mathbb{R}^{\nu} \backslash\{\mathbf{0}\}\right) \times G L(\nu, \mathbb{R}) \mid V_{k+N}=V_{k} M \forall n\right\} .
$$

There are two natural group actions on $\mathcal{W}$. Introduce the group $\mathcal{C}=F u n\left(\mathbb{Z} / N \mathbb{Z}, \mathbb{R}^{\times}\right)$ of periodic sequences of non-zero real numbers, with $(p q)_{m}=p_{m} q_{m}$ for $p, q \in \mathcal{C}$. The groups $G L_{\nu}$ and $\mathcal{C}$ both have natural (commuting) actions on $\mathcal{W}$,

$$
(p, g) \cdot(V, M)=\left(p V g^{-1}, g M g^{-1}\right) \quad p \in \mathcal{C}, g \in G L_{\nu}
$$

The two group actions are not disjoint. For $k \in \mathbb{R}^{\times}$, the actions of $k \in \mathcal{C}$ and $k^{-1} I d \in G L_{\nu}$ have the same effect. To resolve this it is convenient to replace $G L_{\nu}$ by $S L_{\nu}$ and to leave $\mathcal{C}$ intact. It is also natural to restrict $M$ to lie in $S L_{\nu}$. Denote $S L(\nu, \mathbb{R})$ by $G$ and $\operatorname{sl}(\nu, \mathbb{R})$ by $\mathfrak{g}$. 
Let $R \in \wedge^{2} \mathfrak{g}$ and let $C \in S^{2} \mathfrak{g}$. Set $R_{ \pm}=\frac{1}{2}(R \pm C)$. Let $\phi \in F u n(\mathbb{Z} / N \mathbb{Z}, \mathbb{R})$ be an odd periodic function. Denote by $\sigma$ the "discrete sign function", $\sigma_{m}=\operatorname{sgn}(m)$ if $m \neq 0$ and $\sigma_{0}=0$. Define the bracket on $\mathcal{W}$

$$
\left\{\begin{array}{l}
\left\{V_{m}^{1}, V_{n}^{2}\right\}=V_{m} \otimes V_{n}\left[R+\sigma_{m-n}(C+I d \otimes I d)+\phi_{m-n}\right], \\
\left\{V_{m}^{1}, M^{2}\right\}=V_{m}^{1}\left[M^{2} R_{-}-R_{+} M^{2}\right], \\
\left\{M^{1}, M^{2}\right\}=(M \otimes M) R+R(M \otimes M)-M^{1} R_{+} M^{2}-M^{2} R_{-} M^{1} .
\end{array}\right.
$$

Proposition 1.2. The formulae in (6) define a Poisson bracket on $\mathcal{W}$ if and only if $C$ is the Casimir element and if $R$ is a classical r-matrix, i.e. $R$ satisfies the Yang-Baxter equation $\left[R^{12}, R^{13}\right]+$ c.p. $=-\left[C^{12}, C^{13}\right]$.

Proof. The proof is a straightforward check of the Jacobi identity.

Note that $C$ is defined by the property $(g \otimes h) C=C(h \otimes g) \forall g, h \in G$. Alternatively,

$$
(\xi \otimes \eta)(C+I d \otimes I d)=\eta \otimes \xi \quad \forall \xi, \eta \in \mathbb{R}^{\nu} .
$$

$R$ defines the structure of a Poisson Lie group on $G$ by the Sklyanin formula

$$
\left\{g^{1}, g^{2}\right\}=R g \otimes g-g \otimes g R .
$$

Proposition 1.3. The actions of $\mathcal{C}$ and $G$ given by (5) are Poisson actions in the Poisson Lie group sense, when $G$ has the Sklyanin Poisson structure and $\mathcal{C}$ has the zero Poisson structure.

Primes denote shifts of order up to 3 and a superscript with the corresponding number in brackets denotes higher order shifts, thus for $f \in F u n(\mathbb{Z}, \mathbb{R})$,

$$
\begin{aligned}
f^{\prime} & :=(D f) \quad \text { and } \quad f^{(r)}:=\left(D^{r} f\right), \\
\text { or } \quad\left(f^{\prime}\right)_{m} & :=f_{m+1} \quad \text { and } \quad\left(f^{(r)}\right)_{m}:=f_{m+r} .
\end{aligned}
$$

Define the Wronskian $w: \mathcal{W} \rightarrow \mathbb{R}$ by

$$
w(V, M)=\left|V V^{\prime} \ldots V^{(\nu-1)}\right|, \quad \text { i.e. } w_{m}=\left|V_{m} V_{m+1} \ldots V_{m+\nu-1}\right| .
$$

Usually it will be convenient to write $w(V)$ instead of $w(V, M)$.

Proposition 1.4. The map $(V, M) \mapsto M$ is a momentum map for the action of $G$ (in the Poisson Lie group sense, see [L]). The map $(V, M) \mapsto w(V, M)$ is a momentum map for the action of $\mathcal{C}$.

Proof. The first claim follows directly from the second and third formulae in (6). The second is an interpretation of the formula

$$
\left\{w_{m}, V_{n}\right\}=\left(\sigma_{n-m}+\sum_{r=0}^{\nu-1} \phi_{m+r-n}+\sum_{r=1}^{\nu-1} \delta_{m+r-n}\right) w_{m} V_{n}
$$

which is proved in the appendix. 
Remark 1.5. The space $\mathcal{W}$ was introduced in [MS], where it was observed that there were natural actions of the groups $\mathcal{C}$ and $G$ on $\mathcal{W}$. As was explained in that article, the Poisson structure may be determined completely, up to the freedom in the choice of the arbitrary odd periodic function $\phi$, just by the requirement that the actions of $\mathcal{C}$ and $G$ on $\mathcal{W}$ be Poisson. The choice of label $\mathcal{W}$ is inherited from the role this space plays as the space of "wave-functions" when it arises as solutions of some family of linear problems. It is an important and subtle distinction that here $\mathcal{W}$ is viewed as an independent entity and that the linear problems should rather be seen as being generated from $\mathcal{W}$ than the other way about.

It follows from Poisson Lie theory that the set $C^{\infty}(\mathcal{W})^{\mathcal{C}}$ of smooth $\mathcal{C}$-invariant functions on $\mathcal{W}$ is closed with respect to the Poisson bracket (6) , hence that a Poisson structure is automatically induced on the quotient space $\mathcal{W} / \mathcal{C}$ and the projection $\mathcal{W} \rightarrow \mathcal{W} / \mathcal{C}$ is a Poisson map.

Projection from $\mathcal{W}$ to $\mathcal{W} / \mathcal{C}$ is essentially the projection from $\mathbb{R}^{\nu} \backslash\{\mathbf{0}\}$ to $\mathbb{R P}^{\nu-1}$. On the open subset of $\mathbb{R}^{\nu}$ in which the last component is non-zero, we may define the projection from $\mathcal{W}$ to $\mathcal{W} / \mathcal{C}$ in coordinates by first writing $\mathbb{R}^{\nu} \ni V=\chi(v, 1)$ with $v \in \mathbb{R}^{\nu-1}$ and $\chi \in \mathbb{R}^{\times}$. Then the map is given by $\chi(v, 1) \mapsto v$.

In [MS the main focus of interest was in applying Poisson Lie group reduction to obtain a sequence of Poisson projections starting with $\mathcal{W}$ and ending (for the case $\nu=2$ ) with the lattice Virasoro algebra. Here the emphasis will be slightly different and the following result, valid for arbitrary $\nu$, will be an important one.

Proposition 1.6. The Poisson structure obtained by projection $\mathcal{W} \rightarrow \mathcal{W} / \mathcal{C}$ has the form

$$
\left\{v_{m}^{1}, v_{n}^{2}\right\}=\left(v_{m} \otimes v_{n}\right) \cdot R-\sigma_{m-n}\left(v_{m}-v_{n}\right) \otimes\left(v_{m}-v_{n}\right) .
$$

Proof. See appendix.

Here the notation $(a \otimes b) \cdot R$ denotes the projective action of $R$ on $\left(\mathbb{R} \mathbb{P}^{\nu-1} \otimes \mathbb{R} P^{\nu-1}\right)$. Formula (11) must be complemented by suitable representations of $\left\{v_{m}^{1}, M^{2}\right\}$ and $\left\{M^{1}, M^{2}\right\}$, but these will not be needed, so they are not written here. Two properties of the Poisson structure on $\mathcal{W} / \mathcal{C}$ are:

(1) The action of $G$ on $\mathcal{W} / \mathcal{C}$ defined by projectivising the action of $G$ on $\mathcal{W}$ is a Poisson action.

(2) A striking and noteworthy feature of formula (11) is that it does not depend on $\phi$. Crucial use will be made of this in the next sections.

Remark 1.7. From now on we restrict ourselves to the open set of non-degenerate elements in $\mathcal{W}$, for which $w(V, M) \neq 0$.

The following result2 justifies the subsequent interest in "Poisson tensors with a linear dependence on one of the variables"

\footnotetext{
${ }^{2}$ I am grateful to G.Falqui for teaching me this result, which clearly "everybody should know".
} 
Proposition 1.8. Let $M$ be a Poisson manifold with Poisson tensor P. Let $\xi$ be a vector field on $M$. Suppose that $\left(L_{\xi}\right)^{2} P=0$. Then $L_{\xi} P$ is also a Poisson tensor, from which it follows that the pair $\left(P, L_{\xi} P\right)$ defines a bi-hamiltonian structure on $M$.

Proof. As $P$ is Poisson, we have, using the Schouten bracket notation, $[P, P]=0$. Applying $L_{\xi}$ to this condition,

$$
0=L_{\xi}[P, P]=2\left[L_{\xi} P, P\right] .
$$

Applying $L_{\xi}$ again,

$$
0=L_{\xi}\left[L_{\xi} P, P\right]=\left[\left(L_{\xi}\right)^{2} P, P\right]+\left[L_{\xi} P, L_{\xi} P\right]=\left[L_{\xi} P, L_{\xi} P\right] .
$$

It follows from (13) that $L_{\xi} P$ is Poisson and it follows from (12) that $P$ and $L_{\xi} P$ are compatible.

It is worth also giving a qualification of the notion of linearity of a Poisson tensor. Later in this article we look at Poisson structures which are quadratic-plus-linear in terms of the variables chosen to present them. This means some local coordinates $\mathbf{x}$ are chosen for which all Poisson brackets have the form

$$
\left\{x^{i}, x^{j}\right\}=\mathbf{x}^{T} A(i, j) \mathbf{x}+\mathbf{x}^{T} \mathbf{b}(i, j)=\sum_{k} \sum_{l} A(i, j)_{k l} x^{k} x^{l}+\sum_{k} b(i, j)_{k} x_{k},
$$

where for each $(i, j), A(i, j)$ is a constant, symmetric matrix and $\mathbf{b}(i, j)$ is a constant vector. When such a Poisson tensor is said to have a linear dependence on $x^{k}$ for some $k$, it is meant that the $k$ th diagonal entry of each matrix $A(i, j)$ is zero, i.e. that for all $(i, j), A(i, j)_{k k}=0$, and then it is easy to see that the vector field $\xi$, defined by $d x^{l}(\xi)=\delta_{k}^{l}$, satisfies the condition of Proposition 1.8 .

1.1. Operator notation for sequences in $\mathbb{R}$. It proves useful later on to make use of a notation based on the notion that a sequence $K \in F u n(\mathbb{Z}, \mathbb{R})$ can be viewed as the kernel of an operator on $\operatorname{Fun}(\mathbb{Z}, \mathbb{R})$. Let $K_{m} \in \mathbb{R}$ be a sequence of real numbers, then the corresponding operator, also denoted $K$, is defined by the following property

$$
\forall f \in F u n(\mathbb{Z}, \mathbb{R}), \quad(K \cdot f)_{m}=\sum_{n} K_{m-n} f_{n} .
$$

Using this notation, for example, the kernel of the shift operator $D$ is the function $m \mapsto D_{m}=\delta_{m+1}$. The operator notation will be used extensively to treat various conditions on the function $m \mapsto \phi_{m}$ involved in the definition of the Poisson bracket (6).

\section{Polygons in $\mathbb{R}^{2}$}

For $V$ a sequence in $\mathbb{R}^{2}$, clearly, for any $m$, the three vectors $V_{m}, V_{m+1}, V_{m+2}$ are linearly dependent. Assuming that $V$ is a non-degenerate sequence, that is 
to say $\forall n, w_{n} \neq 0$, the linear dependence may be written in the form $V_{m+2}=$ $\mu_{m} V_{m+1}-\rho_{m} V_{m}$. In the more compact notation defined in (8),

$$
V^{\prime \prime}=\mu V^{\prime}-\rho V \text {. }
$$

Moreover, the sequences $\mu$ and $\rho$ evidently depend on the sequence $V$ and this dependence may be given explicitly:

$$
w(V) \mu(V)=\left|V V^{\prime \prime}\right|, \quad w(V) \rho(V)=w(V)^{\prime} .
$$

It is straightforward to check that $\mu$ and $\rho$ are periodic, i.e. $\forall m\left(\mu_{m+N}, \rho_{m+N}\right)=$ $\left(\mu_{m}, \rho_{m}\right)$, and that $\forall g \in G(\mu(V g), \rho(V g))=(\mu(V), \rho(V))$. Hence, as $\operatorname{dim}(\mathcal{W})=$ $2 N+3$ and $\operatorname{dim}(G)=3, \mu$ and $\rho$ are good coordinates on $\mathcal{W} / G$.

An alternative set of coordinates may be defined as follows. For $V$ a quasiperiodic sequence, let $\Gamma(V) \in \mathcal{C}$ be such that $\tilde{V}=\Gamma V$ has constant Wronskian, i.e. $w(\tilde{V}) \equiv$ const. Then $\tilde{V}^{\prime \prime}=u \tilde{V}^{\prime}-\tilde{V}$, where the coefficient of $-\tilde{V}$ is necessarily 1 . If $N$ is odd $\Gamma$ is determined uniquely, whilst for $N$ even, although $\Gamma$ is not defined uniquely, it still exists. Meanwhile $V \mapsto u(V)$ is an invariant of the $\mathcal{C}$-action as well as of the $G$ action and so projection from $\mathcal{W} / G$ to $\mathcal{C} \backslash \mathcal{W} / G$ is implemented directly in the coordinates $(u, \Gamma)$ by forgetting $\Gamma$. The simplest way to implement this is to make the change of variables $(u, \Gamma) \mapsto(S, \gamma)=\left(u u^{\prime}, \Gamma \Gamma^{\prime}\right)$. This results in the expressions

$$
\gamma(V)=\frac{1}{w(V)} \quad \text { and } \quad S(V)=\gamma \gamma^{\prime \prime}\left|V V^{\prime \prime}\right|\left|V V^{\prime \prime}\right|^{\prime}=\frac{\left|V V^{\prime \prime}\right|\left|V V^{\prime \prime}\right|^{\prime}}{\left|V V^{\prime}\right|\left|V V^{\prime}\right|^{\prime \prime}}
$$

for $\gamma$ and $S$, by means of which the Poisson structure on $\mathcal{W} / G$ may be explicitly computed in terms of $(S, \gamma)$. As $w$ generates the action of $\mathcal{C}$ on $\mathcal{W},\left\{\gamma_{m}, S_{n}\right\}$ is zero for all $m$ and $n$, so the Poisson bracket is diagonal in the $(S, \gamma)$ representation. Hence the Poisson projection from $\mathcal{W} / G$ to $\mathcal{C} \backslash \mathcal{W} / G$ is represented by the algebra $\left\{S_{m}, S_{n}\right\}$ and by simply "forgetting" the contribution of $\gamma$.

Whilst this route could be taken and a lattice analogue of the Virasoro algebra could be obtained directly at this stage, this will not be the strategy pursued here, as it would be to miss another interesting aspect of the Poisson analysis of the reductions of $\mathcal{W}$. Some intermediate comments are appropriate however:

(1) It has already been pointed out that even for the case of general $\nu$, the Poisson structure obtained by the projection $\mathcal{W} \rightarrow \mathcal{W} / \mathcal{C}$ is independent of the arbitrary function $\phi$. It follows that the Poisson structure on $\mathcal{C} \backslash \mathcal{W} / G$ must be independent of $\phi$.

(2) Although it was convenient in the previous paragraph to propose the presentation of the Poisson structure on $\mathcal{C} \backslash \mathcal{W} / G$ via the variables $(S, \gamma)$, it is evident that the same splitting phenomenon will take place in the variables $(u, \Gamma)$ and so a representation of the reduced Poisson algebra will also exist in terms of the variables $u$. Indeed if $N$ is odd the map $u \mapsto S$ is invertible and such a representation may be obtained directly from the formula for $\left\{S_{m}, S_{n}\right\}$. 
2.1. Reduction via the Dirac method. Having observed that the reduction $\mathcal{W} \rightarrow \mathcal{C} \backslash \mathcal{W} / G$ is a Poisson reduction and that it can be presented explicitly by computing the Poisson brackets in terms of the coordinates $(S, \gamma)$ on $\mathcal{W} / G$, it is interesting to perform the reduction by a different route, using the Dirac constraint method with the variables $(\mu, \rho)$ on $\mathcal{W} / G$. Of course $\left.\mu\right|_{\rho \equiv 1}$ is the same as $u$.

Working in terms of the variables $(\mu, \rho)$ the relation in (15) is recognised to be the standard Lax-matrix for the Toda system. More specifically, it will be the periodic Toda lattice due to the periodicity inherent in the setting chosen here. We undoubtedly are about to uncover Poisson structures associated with the same space and might expect that they have something to do with the Toda lattice. As we shall see, this turns out indeed to be the case.

Using the explicit expressions in (16), the representation of the Poisson structure on $\mathcal{W} / G$ is computed explicitly in terms of $(\mu, \rho)$. The result is the following algebra

$$
\left\{\begin{aligned}
\left\{\mu_{m}, \mu_{n}\right\}= & \left(2 \phi_{m-n}-\phi_{m-n+1}-\phi_{m-n-1}\right. \\
& \left.-\delta_{m-n+1}+\delta_{m-n-1}\right) \mu_{m} \mu_{n}+2 \delta_{m-n+1} \rho_{n}-2 \delta_{m-n-1} \rho_{m} \\
\left\{\mu_{m}, \rho_{n}\right\}= & \left(\phi_{m-n}+\phi_{m-n+1}-\phi_{m-n-1}-\phi_{m-n+2}\right. \\
- & \left.\delta_{m-n}+\delta_{m-n-1}+\delta_{m-n+1}-\delta_{m-n+2}\right) \mu_{m} \rho_{n} \\
\left\{\rho_{m}, \rho_{n}\right\}= & \left(2 \phi_{m-n}-\phi_{m-n+2}-\phi_{m-n-2}\right. \\
- & \left.\delta_{m-n+2}+\delta_{m-n-2}\right) \rho_{m} \rho_{n}
\end{aligned}\right.
$$

This Poisson structure depends on $\phi$, but we know in advance that the structure obtained by constraint to $\rho \equiv 1$ will not depend on $\phi$. In fact there are two special choices for $\phi$ which present themselves as deserving attention. One choice is useful for extracting the formula for the Poisson structure on $\mathcal{C} \backslash \mathcal{W} / G$ in terms of $u=\left.\mu\right|_{\rho \equiv 1}$, rather than in terms of $S$, reproducing the result of [FRS]. The other choice will give rise to the Poisson structure of the Toda lattice.

2.1.1. $\phi$ chosen to diagonalise the Poisson structure. In applying the Dirac constraint method it will be convenient if we are able to make use of the freedom in the choice of $\phi$ in order to make the bracket $\left\{\mu_{m}, \rho_{n}\right\}$ identically zero. The constrained Poisson bracket will then be given by the formula for $\left\{\mu_{m}, \mu_{n}\right\}$ in (17) with $\phi$ replaced by this special choice. The essential question here is Does there exist a solution to the following difference equation?

$$
\phi_{m}+\phi_{m+1}-\phi_{m-1}-\phi_{m+2}-\delta_{m}+\delta_{m-1}+\delta_{m+1}-\delta_{m+2}=0 .
$$

A convenient way to think of (18) is to interpret $\phi$ as the kernel of an operator, according to (14). (18) may then be written as the operator equation

$$
\left(D^{3}-D^{2}-D+1\right) \phi=-D^{3}+D^{2}-D+1
$$


which may be easily solved to give

$$
\phi=\frac{1+D^{2}}{1-D^{2}}
$$

as an operator. As a function, $\phi$ is the kernel of the operator ${ }^{3}$ in (19). What happens now is that in fact for this choice of $\phi$, the Poisson bracket $\left\{\rho_{m}, \rho_{n}\right\}$ also vanishes, so that $\rho$ is a Casimir. This implies the "first-class constraint" condition in [FRS], although $\rho$ being a Casimir is a stronger condition. It is now a straightforward exercise to compute the coefficient involved in the bracket $\left\{\mu_{m}, \mu_{n}\right\}$ of (17). Thus, using the operator presentation of $\phi$, for which $2 \phi_{m}-\phi_{m+1}-\phi_{m-1}-\delta_{m+1}+\delta_{m-1} \sim$ $D^{-1}\left[2 D-1-D^{2}\right] \phi-D+D^{-1}$

$$
\begin{aligned}
-D^{-1}\left[(1-D)^{2} \phi-D^{2}+1\right] & =-D^{-1}(1-D)[(1-D) \phi-1-D] \\
& =-D^{-1}(1-D)\left[(1+D)^{-1}\left(1+D^{2}\right)-1-D\right] \\
& =D^{-1}(D-1)(D+1)^{-1}\left[1+D^{2}-(1+D)^{2}\right] \\
& =2 \frac{1-D}{1+D} .
\end{aligned}
$$

We obtain the Poisson bracket on $\mathcal{C} \backslash \mathcal{W} / G$ in the variable $u=\left.\mu\right|_{\rho \equiv 1}$,

$$
\left\{u_{m}, u_{n}\right\}=\pi_{m-n} u_{m} u_{n}+\delta_{m-n+1}-\delta_{m-n-1}
$$

where $\pi \in F u n(\mathbb{Z} / N \mathbb{Z}, \mathbb{R})$ is the kernel of the operator $(1-D)(D+1)^{-1}$. Here the bracket in (16) has been divided by 2. Alternatively, the Poisson tensor may be written in operator form,

$$
P(u)=u\left(\frac{1-D}{1+D}\right) u+D-D^{-1} .
$$

This is the same as the Poisson tensor obtained in [FRS].

2.1.2. Choice of $\phi$ adapted to the Toda lattice interpretation. Let us choose $\phi$ so that the Poisson bracket depends linearly on $\mu$. As the only quadratic term in $\mu$ is in the $\left\{\mu_{m}, \mu_{n}\right\}$ bracket, we should look for a solution of the difference equation

$$
2 \phi_{m}-\phi_{m+1}-\phi_{m-1}-\delta_{m+1}+\delta_{m-1}=0 .
$$

In operator form, we have the equation for $\phi$

$$
(D-1)^{2} \phi+D^{2}-1=0
$$

which has the solution

$$
\phi=\frac{1+D}{1-D} .
$$

\footnotetext{
${ }^{3}$ Some care must be taken in the interpretation of formula (19). It was specified that $m \mapsto \phi_{m}$ has to be a periodic function. This means that the shift operator $D$ has to be viewed here as the periodic shift operator, i.e. such that $D^{\nu}=I d$. However, in all equations which make use of the formula (19) this subtlety is not an issue as the periodic operator will always be applied to a periodic sequence, for which there is no difference between the standard shift operator and the periodic one.
} 
Using this choice for $\phi$ we should now evaluate the coefficients appearing in the other Poisson brackets of (17). In operator form, for the $\left\{\mu_{m}, \rho_{n}\right\}$ bracket we must compute

$$
\begin{aligned}
-D^{-1}\left[\left(D^{3}-D^{2}-D+1\right) \phi+\right. & \left.D^{3}-D^{2}+D-1\right] \\
& =-D^{-1}(D-1)\left[\left(D^{2}-1\right) \phi+\left(D^{2}+1\right)\right] \\
& =D^{-1}(D-1)\left[(1+D)^{2}-\left(1+D^{2}\right)\right] \\
& =2(D-1) .
\end{aligned}
$$

For the $\left\{\rho_{m}, \rho_{n}\right\}$ bracket we must compute

$$
\begin{aligned}
D^{-2}\left[1-D^{4}-\left(1-D^{2}\right)^{2} \phi\right] & =D^{-2}\left(1-D^{2}\right)\left[\left(1+D^{2}\right)-(1+D)^{2}\right] \\
& =-2 D^{-1}\left(1-D^{2}\right) \\
& =2\left(D-D^{-1}\right) .
\end{aligned}
$$

Putting these together, we obtain, for the choice of $\phi$ in (23), dividing the right-hand side of (17) by 2 ,

$$
\begin{aligned}
& \left\{\mu_{m}, \mu_{n}\right\}=\delta_{m-n+1} \rho_{n}-\delta_{m-n-1} \rho_{m} \\
& \left\{\mu_{m}, \rho_{n}\right\}=\left(\delta_{m-n+1}-1\right) \mu_{m} \rho_{n} \\
& \left\{\rho_{m}, \rho_{n}\right\}=\left(\delta_{m-n+1}-\delta_{m-n-1}\right) \rho_{m} \rho_{n} .
\end{aligned}
$$

In operator form, this Poisson structure is given by

$$
\left(\begin{array}{c}
\dot{\mu} \\
\dot{\rho}
\end{array}\right)=\left(\begin{array}{cc}
D \rho-\rho D^{-1} & \mu(D-1) \rho \\
\rho\left(1-D^{-1}\right) \mu & \rho\left(D-D^{-1}\right) \rho
\end{array}\right) \cdot\left(\begin{array}{c}
\delta_{\mu} H \\
\delta_{\rho} H
\end{array}\right)
$$

which is the "second Poisson structure" for the Toda lattice.

It is worth pointing out that the "lifted version" of the standard Toda flow to the space $\mathcal{W}$ can be computed as the Hamiltonian flow with Hamiltonian $H(V)=$ $\sum_{m} \mu_{m}(V)$. The result is

$$
\dot{V}=D^{-1}(\rho V) \quad \text { or } \quad \dot{V}_{m}=\frac{\left|V_{m} V_{m+1}\right|}{\left|V_{m-1} V_{m}\right|} V_{m-1} .
$$

\subsubsection{Faddeev-Takhtajan-Volkov obtained as constrained Toda.}

Corollary 2.1. Applying the constraint $\rho \equiv 1$ to the Poisson structure in (24, 25) results in the lattice Virasoro structure (20, 21) of [FRS].

Proof. This follows directly from Proposition 1.6. A demonstration of the result is given in the Appendix.

To close this section let us state the result already alluded to and proven indeed in [FRS, 
Proposition 2.2. In the variables $S$ on $\mathcal{C} \backslash \mathcal{W} / G$, given by the change of variables $u \mapsto S=u u^{\prime}$, the Poisson structure (21) is represented by

$$
\begin{aligned}
P(S)=D S+ & S D-D^{-1} S-S D^{-1}+S D^{-1} S-S D S \\
& +S D S^{-1} D S-S D^{-1} S^{-1} D^{-1} S
\end{aligned}
$$

Proof. A demonstration of this result is also given in the Appendix.

2.1.4. A connection with the Dressing Chain of Veselov and Shabat. The description of coordinates on $\mathcal{C} \backslash \mathcal{W} / G$ can be modified and generalised to incorporate a result from the paper of Veselov and Shabat [VS]. Suppose that $\beta$ is a fixed periodic sequence in $\mathbb{R}^{\times}$and let $B$ be a sequence for which $B^{\prime}=\beta B$ (i.e. $B_{m}=$ $\left.\beta_{m-1} \beta_{m-2} \cdots \beta_{0}\right)$. For $V$ a quasi-periodic sequence, let $\Gamma(V) \in \mathcal{C}$ be such that the Wronskian of $\tilde{V}=\Gamma V$ has the property, $\frac{w(\tilde{V})^{\prime}}{w(\tilde{V})} \equiv \beta$. We have then $\tilde{V}^{\prime \prime}=u \tilde{V}^{\prime}-\beta \tilde{V}$. $u$ is an invariant of the $\mathcal{C}$-action as well as of the $G$ action and so projection from $\mathcal{W} / G$ to $\mathcal{C} \backslash \mathcal{W} / G$ is implemented directly in the coordinates $(u, \Gamma)$ by forgetting $\Gamma$. The simplest way to implement this is to make the change of variables $(u, \Gamma) \mapsto(S, \gamma)=\left(\beta^{\prime-1} u u^{\prime}, \Gamma \Gamma^{\prime}\right)$. This results in the expressions

$$
\gamma(V)=\frac{B}{w(V)} \quad \text { and } \quad S(V)=\frac{\left|V V^{\prime \prime}\right|\left|V V^{\prime \prime}\right|^{\prime}}{\left|V V^{\prime}\right|\left|V V^{\prime}\right|^{\prime \prime}}
$$

for $\gamma$ and $S$, by means of which the Poisson structure on $\mathcal{W} / G$ is explicitly computed in terms of $(S, \gamma)$. As in the special case $\beta \equiv 1$ already discussed, the Poisson projection from $\mathcal{W} / G$ to $\mathcal{C} \backslash \mathcal{W} / G$ is represented by the algebra $\left\{S_{m}, S_{n}\right\}$ and by simply "forgetting" the contribution of $\gamma$. The formulae in (20) and (21) are modified in this more general case by constraining to $\rho \equiv \beta$ instead of to $\rho \equiv 1$.

Note 2.3. This means that the lattice Virasoro algebra made its first appearance, in the more general form with $\beta$ an arbitrary periodic sequence, in the article of Veselov and Shabat, although it seems not to have been recognised as being equivalent to the Faddeev-Takhtajan-Volkov structure

\section{Polygons in $\mathbb{R}^{3}$}

It is instructive to look at the $\nu=3$ case in the same detail. For $V$ a sequence in $\mathbb{R}^{3}$, for any $m$, the four vectors $V_{m}, V_{m+1}, V_{m+2}, V_{m+3}$ are linearly dependent. Assuming that $V$ is a non-degenerate sequence, the linear dependence may be written in the form $V_{m+3}=a_{m} V_{m+2}-b_{m} V_{m+1}+\rho_{m} V_{m}$. In more compact notation, we have

$$
V^{\prime \prime \prime}=a V^{\prime \prime}-b V^{\prime}+\rho V \text {. }
$$

The sequences $a, b$ and $\rho$ depend on the sequence $V$ and they may be expressed explictly:

$$
w(V) a(V)=\left|V V^{\prime} V^{\prime \prime \prime}\right|, \quad w(V) b(V)=\left|V V^{\prime \prime} V^{\prime \prime \prime}\right|, \quad w(V) \rho(V)=w(V)^{\prime} .
$$

\footnotetext{
${ }^{4} \mathrm{I}$ am grateful to A.Veselov for discussions in which this observation came to light.
} 
$a, b$ and $\rho$ are periodic and $\forall g \in G(a(V g), b(V g), \rho(V g))=(a(V), b(V) \rho(V))$. Hence, $\operatorname{as} \operatorname{dim}(\mathcal{W})=3 N+\operatorname{dim}(G),(a, b, \rho)$ are good coordinates on $\mathcal{W} / G$.

Using the expressions in (29), we may compute the representation of the Poisson structure on $\mathcal{W} / G$ in terms of $(a, b, \rho)$ explicitly:

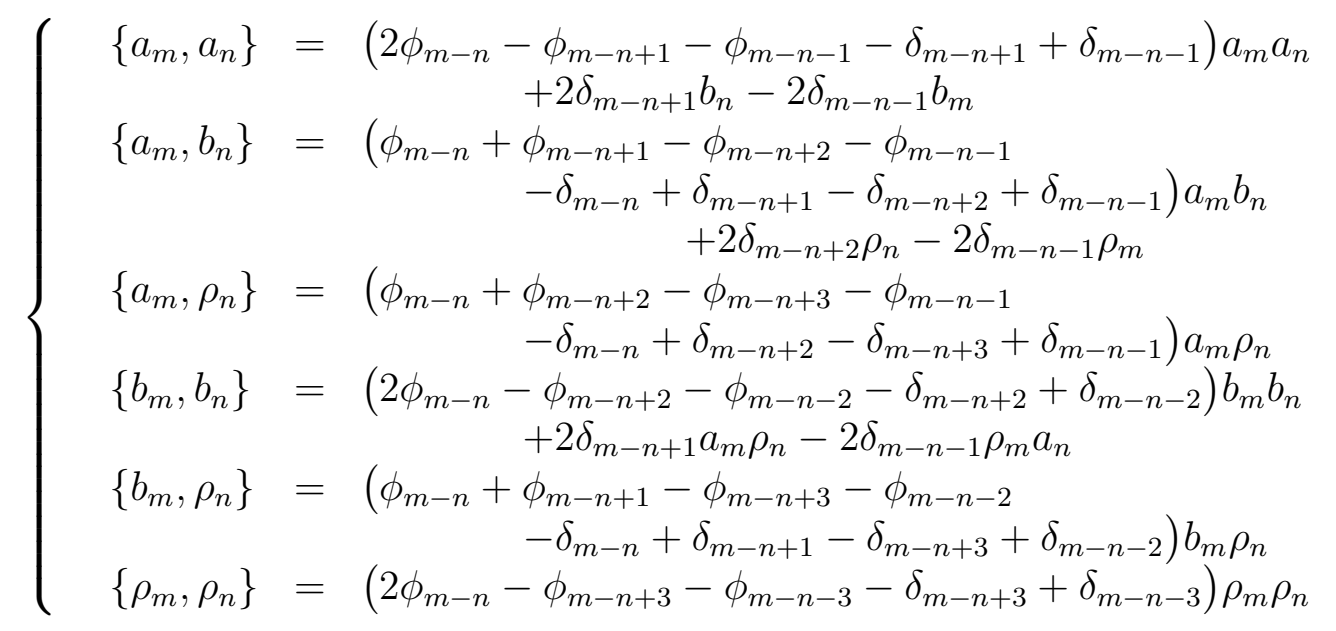

3.1. Choices for the function $\phi$. Constraint of the algebra (30) to $\rho \equiv 1$ (or to any fixed periodic sequence $\rho \equiv \beta$ in $\mathbb{R}^{\times}$) is guaranteed to be independent of $\phi$, due to Proposition 1.6. There are three natural special choices for $\phi$ which present themselves for closer inspection. The first is the one for which $\rho$ is a Casimir of (30). The second is the one for which the Poisson tensor depends linearly on the variable $a$. The third is the one for which the Poisson tensor depends linearly on the variable $b$.

Remark 3.1. It is not obvious that there exists a choice of $\phi$ for which $\rho$ is a Casimir. That this turns out to be possible implies the crucial result in [SS that it is possible to fix their r-matrix in such a way for the constraints to be first-class. In the present context it may be found by straightforward computation that imposing say $\left\{a_{m}, \rho_{n}\right\}=0$ implies both $\left\{b_{m}, \rho_{n}\right\}=0$ and $\left\{\rho_{m}, \rho_{n}\right\}=0$. Making the resulting choice for $\phi$ is equivalent to singling out the r-matrix in [SS].

3.1.1. $\phi$ chosen so that $\rho$ is a Casimir. Let us look for $\phi$ in (30) to force the brackets $\left\{a_{m}, \rho_{n}\right\}$ to vanish. The equation for $\phi$ is

$$
\left(-D^{4}+D^{3}+D-1\right) \phi=\left(D^{4}-D^{3}+D-1\right)
$$

which has the solution

$$
\phi=\frac{1+D^{3}}{1-D^{3}}
$$

The following proposition may be checked by substitution of (31) in (30).

Proposition 3.2. When the function $\phi$ is the kernel of the operator in (31) $\rho$ is a Casimir function of the Poisson structure defined by (30). 
It follows that the reduced Poisson structure on $\mathcal{C} \backslash \mathcal{W} / G$ is given in local coordinates $(a, b)$, with $\rho \equiv 1$, by the relevant brackets in (30) in which $\phi$ is the operator in (31):

$$
\left(\begin{array}{c}
\dot{a} \\
\dot{b}
\end{array}\right)=P_{0}(a, b) \cdot\left(\begin{array}{c}
\delta_{a} H \\
\delta_{b} H
\end{array}\right),
$$

with

$$
P_{0}(a, b)=\left(\begin{array}{cc}
a\left(1+D+D^{2}\right)^{-1}\left(1-D^{2}\right) a & a\left(1+D+D^{2}\right)^{-1}\left(D-D^{2}\right) b \\
+D b-b D^{-1} & +D^{2}-D^{-1} \\
& \\
b\left(1+D+D^{2}\right)^{-1}(1-D) a & b\left(1+D+D^{2}\right)^{-1}\left(1-D^{2}\right) b \\
+D-D^{-2} & +a D-D^{-1} a
\end{array}\right) .
$$

3.1.2. $\phi$ chosen so that the Poisson tensor depends linearly on a. Choosing

$$
\phi=\frac{1+D}{1-D}
$$

solves the equation

$$
\left(2-D-D^{-1}\right) \phi-D+D^{-1}=0
$$

and forces the dependence on $a$ to be linear. The Poisson bracket (29) with $\phi$ substituted from (33) is

$$
\left(\begin{array}{c}
\dot{a} \\
\dot{b} \\
\dot{\rho}
\end{array}\right)=P_{1}(a, b, \rho) \cdot\left(\begin{array}{c}
\delta_{a} H \\
\delta_{b} H \\
\delta_{\rho} H
\end{array}\right)
$$

with $P_{1}(a, b, \rho)=$

$$
\left(\begin{array}{ccc}
D b-b D^{-1} & a(D-1) b & a\left(1-D^{2}\right) \rho \\
& +D^{2} \rho-\rho D^{-1} & \\
b\left(1-D^{-1}\right) a & b\left(D-D^{-1}\right) b & b\left(D-D^{-1}\right)(D+1) \rho \\
+D \rho-\rho D^{-2} & +a D \rho-\rho D^{-1} a & \\
\rho\left(D^{-2}-1\right) a & \rho\left(1+D^{-1}\right)\left(D-D^{-1}\right) b & \rho\left(D^{2}+D-D^{-1}-D^{-2}\right) \rho
\end{array}\right) .
$$

3.1.3. $\phi$ chosen so that the Poisson tensor depends linearly on b. Choosing

$$
\phi=\frac{1+D^{2}}{1-D^{2}}
$$

solves the equation

$$
\left(1+D-D^{2}-D^{-1}\right) \phi-1+D-D^{2}+D^{-1}=0
$$


and forces the dependence on $b$ to be linear. The Poisson bracket (29) with $\phi$ substituted from (35) is

$$
\left(\begin{array}{c}
\dot{a} \\
\dot{b} \\
\dot{\rho}
\end{array}\right)=P_{2}(a, b, \rho) \cdot\left(\begin{array}{c}
\delta_{a} H \\
\delta_{b} H \\
\delta_{\rho} H
\end{array}\right)
$$

with $P_{2}(a, b, \rho)=$

$$
\left(\begin{array}{ccc}
a \frac{1-D}{1+D} a+D b-b D^{-1} & D^{2} \rho-\rho D^{-1} & a D \frac{D-1}{D+1} \rho \\
D \rho-\rho D^{-2} & a D \rho-\rho D^{-1} a & b(D-1) \rho \\
\rho D^{-1} \frac{D-1}{1+D} a & \rho\left(1-D^{-1}\right) b & \rho \frac{D-1}{D+1}\left(D+1+D^{-1}\right) \rho
\end{array}\right)
$$

3.2. Discussion of $\nu=3$ examples. The Poisson structures presented in equations (34) and in (36) are the same as the ones related to different "extended Toda" systems, see for example [C]. This means that by seeing those structures in the context of the present article, they are clearly compatible with one another, as their sum is the special case of (29) for which $\phi$ is the sum of the kernels of the operators in (33) and (35), which is just some other odd function.

On the other hand, the compatibility of the two Poisson structures (34) and (36) in the usual context of extended Toda systems seems not only surprising, but totally inappropriate as it appears to amount to the comparison of two structures defined on different spaces: that is, $P_{1}$ is naturally defined on the set of difference operators of the form $\left\{D+u_{0}+u_{-1} D^{-1}+u_{-2} D^{-2}\right\}$ and $P_{2}$ is naturally defined on the set of difference operators of the form $\left\{D^{2}+u_{1} D+u_{0}+u_{-1} D^{-1}\right\}$. In the setting of the present article these two spaces are just the same, but in the standard setting of Toda systems they are not.

The Poisson structures $P_{1}$ and $P_{2}$ in (34) and in (36) were obtained in [C] by applying a formula in [LP, OR] for which the two extended Toda spaces are not treated in the same way at all.

\section{A Result FOR THE GENERAL $\nu$ CASE}

We observe that the different choices of $\phi$ of especial interest have a natural form. That is, for $\nu=2$ we make the choices

$$
\phi=\frac{1+D^{2}}{1-D^{2}} \quad \text { and } \quad \phi=\frac{1+D}{1-D}
$$

and for $\nu=3$ the special choices are

$$
\phi=\frac{1+D^{3}}{1-D^{3}}, \quad \phi=\frac{1+D^{2}}{1-D^{2}}, \quad \text { and } \quad \phi=\frac{1+D}{1-D} .
$$

We may imagine that the obvious pattern shows itself in the case of general $\nu$. First of all we need to draw attention to a particularly useful feature of the choices 
above. In each case, the first choice is the one which makes the reduction to the space $\mathcal{C} \backslash \mathcal{W} / G$ explicit, whilst the others have the property that they render the Poisson structure on $\mathcal{W} / G$ linear in one or other of the variables. This property of linearity is important in the context of bi-hamiltonian systems as it guarantees the possibility to find a vector field satisfying the conditions of Proposition 1.8 and so to deform the Poisson structure in such a way to obtain another one which is necessarily compatible with it. We may note that not only does this observation apply to the formulae (25, 34, 36), but it corresponds to standard known results for Toda and extended Toda systems, see for example [C].

Let us look at what happens in the general case. First of all, observing that the $\nu+1$ vectors $\mathbb{R}^{\nu} \ni V, V^{\prime}, V^{\prime \prime}, \ldots, V^{(\nu)}$ obey some linear dependence relation and that the nondegeneracy condition $w(V) \neq 0$ allows us to assume the coefficient of $V^{(\nu)}$ in this relation to be 1 , we may write

$$
V^{(\nu)}-a^{(\nu-1)} V^{(\nu-1}+\cdots+(-1)^{(\nu-r)} a^{(\nu-r)} V^{(\nu-r)}+\cdots+(-1)^{\nu} a^{(0)} V=0 .
$$

Hence the quasi-periodic sequence $V \in \mathcal{W}$ engenders the element $L=D^{\nu}-$ $a^{(\nu-1)} D^{\nu-1}+\cdots+(-1)^{\nu-1} a^{(1)} D+(-1)^{\nu} a^{(0)} \in \mathcal{S}_{\nu}$ and the fields $\left(a^{(0)}, a^{(1)}, \ldots, a^{(\nu-1)}\right)$ are a good set of coordinates on $\mathcal{W} / G$. Let us set $\mathbf{a}=\left(a^{(0)}, a^{(1)}, \ldots, a^{(\nu-1)}\right)$. For $k=1, \ldots, \nu-1$, define the functions $\alpha^{(k)}$ on $\mathcal{W}$ by

$$
\alpha^{(k)}=\left|V \ldots V^{(\nu)}\right|^{\vee} \text {, i.e. } \alpha_{m}^{(k)}=\left|V_{m} \ldots V_{m+k-1} V_{m+k+1} \ldots V_{m+\nu}\right|
$$

where the symbol $\stackrel{k}{\vee}$ on the determinant means that the $k$ th term is not present, as is clear in the second expression. It is easy to check that

$$
\text { For } 1 \leq k \leq \nu-1, \quad a^{(k)}=\frac{\alpha^{(k)}}{w} \quad \text { and } \quad a^{(0)}=\frac{w^{\prime}}{w} .
$$

For a general value of $\nu$ a generalisation of the results of Sections 2 and 3 provides positive answers to the questions

For $k \in\{1, \ldots, \nu-1\}$, is there a choice of $\phi$ such that the Poisson tensor $P_{\phi}(\mathbf{a})$ on $\mathcal{W} / G$, when represented in the coordinates $\mathbf{a}$, is linear in the coordinate $a^{(k)}$ : so that $\frac{d^{2}}{d t^{2}} P\left(a^{(0)}, \ldots, a^{(k)}+t, \ldots, a^{(\nu-1)}\right)=0$ ?

Is there a choice of $\phi$ such that $a^{(0)}$ is a Casimir?

in the form of the following theorem, whose proof is given in Appendix B.

Theorem 4.1. For the family of $\phi$-dependent Poisson brackets on $\mathcal{S}_{\nu}=\mathcal{W} / G$, with respect to the natural coordinates a on $\mathcal{S}_{\nu}$, given by $\mathcal{S}_{\nu} \ni L=D^{\nu}-a^{(\nu-1)} D^{\nu-1}+$ $\cdots+(-1)^{\nu-1} a^{(1)} D+(-1)^{\nu} a^{(0)}$

(i) the choice

$$
\phi^{(0)}:=\frac{1+D^{\nu}}{1-D^{\nu}}
$$


forces $a^{(0)}$ to be a Casimir;

(ii) for $0<k<\nu$, the choice

$$
\phi^{(k)}:=\frac{1+D^{\nu-k}}{1-D^{\nu-k}}
$$

forces the Poisson bracket to have linear dependence on $a^{(k)}$.

The following corollary tells us that we can introduce a spectral parameter in a straightforward way for any of the choices $\phi=\phi^{(k)}$ for $0 \leq k \leq \nu$

Corollary 4.2. A spectral parameter $\lambda$ can be introduced as follows: With the choice $\phi=\phi^{(0)}$ and $\beta$ any fixed periodic sequence, $\rho \equiv \beta$ can be replaced by $\rho \equiv \beta+\lambda$. With the choice $\phi=\phi^{(k)}$, for $0<k<\nu, a^{(k)}$ can be replaced by $a^{(k)}+\lambda$.

\section{Conclusion and Perspectives}

As was shown in $[\mathrm{MS}$, introducing a Poisson structure on the space $\mathcal{W}$ with the property that the actions of $\mathcal{C}$ and $G$ on $\mathcal{W}$ be Poisson is a natural setting for the Poisson description of the space $\mathcal{S}_{\nu}$, by the identification $\mathcal{S}_{\nu}=\mathcal{W} / G$. In fact we have proceeded on the basis of an initial family of (compatible) Poisson structures on $\mathcal{W}$ parametrised by an arbitrary odd function $\phi$ appearing in the formula for the original Poisson bracket on $\mathcal{W}$. Consequently there is a family of Poisson structures on $\mathcal{S}_{\nu}=\mathcal{W} / G$, still parametrised by the arbitrary odd function $\phi$. It has been shown however that the Poisson structure on $\mathcal{C} \backslash \mathcal{W}$, obtained by reducing the one on $\mathcal{W}$, is independent of $\phi$. It follows that the projection $\Pi: \mathcal{W} / G \rightarrow \mathcal{C} \backslash \mathcal{W} / G$ takes the $\phi$-dependent family of Poisson structures to a single $\phi$-independent structure. The map $\Pi$ is represented in the space $\mathcal{S}_{\nu}$ by imposing the constant-Wronskian constraint, $u_{0} \equiv(-1)^{\nu}$, where $\mathcal{S}_{\nu}=\left\{L=D^{\nu}+u_{\nu-1} D^{\nu-1}+\cdots+u_{1} D+u_{0}\right\}$ and therefore the Poisson structure can be discovered by applying Dirac's method of constraints. It has been shown how the freedom in the choice of $\phi$ for the result of applying the projection $\Pi$ may be used to simplify the computations of the reduction. In the cases $\nu=2,3$ it has been noticed that other special choices for the function $\phi$ lead to the (second) Poisson structures for Toda and extended Toda systems. A simple corollary is that a constraint of the second Toda Poisson bracket yields the lattice Virasoro Poisson bracket and that the same thing happens for the extended Toda systems and the higher rank versions of lattice Virasoro, known as lattice W-algebras.

It seems undeniable that the point of view presented first in [MS] and here developed further cannot fail to be a useful one, extending as it manifestly does, the $s l_{\nu}$ cases of results presented in [FRS] and [SS]. It will be interesting to discover how to extend the approach taken here to encapsulate all of the cases in [SS] covering as they do all the classical root systems and not just the ones of type $A_{n}$. It is expected that such a generalisation may have useful applications for the construction of examples of interesting new Frobenius manifolds, [D]: see for example the articles [CDZ, DZ].

Perhaps the most interesting outcome of the analysis developed in the present article is the understanding of how a spectral parameter can be introduced. It is 
expected that this could lead to a constructive method for generating integrable flows. It would be interesting to understand how the simple dependence of the fields $a^{(k)}$ on the spectral parameter $\lambda$ for different choices of $\phi$ are reflected in some more complicated dependence on $\lambda$ of the wave-functions $V \in \mathcal{W}$.

A tantalising problem of some interest to the author is to discover the link between a result of the article OST and the Poisson structure on $\mathcal{W}$. It seems inconceivable that such a link should not exist, but so far it has not proved possible to identify one. The original hope was that the Poisson structure found in [OST] might prove to be compatible with the one on $\mathcal{C} \backslash \mathcal{W} / G$, but this is not the case. This link is especially interesting because the integrable system described in [OST] does have a Lax representation depending on a spectral parameter.

The Poisson structure presented in the formula (6) and in Proposition 1.2 is not an original one. It is a representation of the so-called "exchange algebra". The quantum version of the exchange algebra, from which the Poisson case can be extracted as a classical limit, is explained in the lecture notes [F] of Faddeev. It appeared already in the article [S1] and has been made use of in many contexts since then, see for example [BFP]. More notably it was also used in a form almost the same as that exploited here, by Babelon, especially in [B]. Indeed the only thing added here to Babelon's work is the recognition of the role of Poisson Lie groups and of the additional function $\phi$.

\section{Appendix A: Missing proofs}

6.1. Proof of Proposition 1.4. The claim was that $w$ defines a momentum map for the action of $\mathcal{C}$ on $\mathcal{W}$. The following lemmas are used in the proof.

Lemma 6.1. $\sigma_{m+k}=\sigma_{m}+\delta_{m}+2\left[\delta_{m+1}+\cdots+\delta_{m+k-1}\right]+\delta_{m+k}$.

Proof. The case $k=1, \sigma_{m+1}-\sigma_{m}=\delta_{m}+\delta_{m+1}$ is easy to check. The general case may then be proved by induction. Equivalently, the operator whose kernel is the function $m \mapsto \sigma_{m}$ is the Cayley transform of $D$,

$$
\sigma=\frac{D+1}{D-1}
$$

from which the same formula is found directly.

Lemma 6.2. Let $\xi_{1}, \xi_{2} \ldots, \xi_{\nu} \in \mathbb{R}^{\nu}$ and $X \in \mathfrak{g}$. Then $\sum_{k=1}^{\nu}\left|\xi_{1} \ldots\left(X \xi_{k}\right) \ldots \xi_{\nu}\right|=0$.

Proof. For $g(t)=\exp (t X)$, writing $\tilde{\xi}_{k}(t)=g(t) \xi_{k} \forall k$,

$$
\begin{aligned}
\sum_{k=1}^{\nu}\left|\xi_{1} \ldots \xi_{k-1}\left(X \xi_{k}\right) \xi_{k+1} \ldots \xi_{\nu}\right| & =\left.\frac{d}{d t}\right|_{t=0}\left|\tilde{\xi}_{1}(t) \ldots \tilde{\xi}_{k}(t) \ldots \tilde{\xi}_{\nu}(t)\right| \\
& =\left.\frac{d}{d t}\right|_{t=0}|g(t)|\left|\xi_{1} \ldots \xi_{\nu}\right|=0 .
\end{aligned}
$$


Proof of Proposition 1.4

Writing $R=\sum_{i} X_{i} \otimes Y_{i}$ and $C=\sum_{j} P_{j} \otimes Q_{j}$ for suitable $X_{i}, Y_{i}, P_{j}, Q_{j} \in \mathfrak{g}$, we may compute

$$
\begin{aligned}
\left\{w_{m}, V_{n}\right\}=\sum_{k=0}^{\nu-1}\left(\sum_{i}\left|V_{m} \ldots V_{m+k-1}\left(V_{m+k} X_{i}\right) V_{m+k+1} \ldots V^{(\nu-1)}\right| V_{n} Y_{i}\right. \\
\quad-\sigma_{m+k-n}\left|V_{m} \ldots V_{m+k-1} V_{n} V_{m+k+1} \ldots V^{(\nu-1)}\right| V_{m+k} \\
\left.\quad+\phi_{m+k-n}\left|V_{m} \ldots V_{m+k} \ldots V^{(\nu-1)}\right| V_{n}\right) \\
=\sum_{i}\left(\sum_{k=0}^{\nu-1}\left|V_{m} \ldots\left(V_{m+k} X_{i}\right) \ldots V^{(\nu-1)}\right|\right) V_{n} Y_{i} \\
\quad-\sum_{k=0}^{\nu-1} \sigma_{m+k-n}\left|V_{m} \ldots V_{m+k-1} V_{n} V_{m+k+1} \ldots V^{(\nu-1)}\right| V_{m+k} \\
\quad+\left(\sum_{k=0}^{\nu-1} \phi_{m+k-n}\right) w_{m} V_{n}
\end{aligned}
$$

where use has been made of the property (7). The first term is zero by Lemma 6.2. Using Lemma 6.1, the general term in the second sum is $\left(\sigma_{m-n}+\delta_{m-n}+2\left[\delta_{m-n+1}+\right.\right.$ $\left.\left.\cdots+\delta_{m-n+k-1}\right]+\delta_{m-n+k}\right)\left|V_{m} \ldots V_{m+k-1} V_{n} V_{m+k+1} \ldots V^{(\nu-1)}\right|$. Hence

$$
\begin{aligned}
\sigma_{m-n+k} \mid V_{m} & \ldots V_{n} \ldots V^{(\nu-1)}\left|V_{m+k}=\left(\sigma_{m-n}+\delta_{m+k-n}\right)\right| V_{m} \ldots V_{n} \ldots V^{(\nu-1)} \mid V_{m+k} \\
& =\sigma_{m-n}\left|V_{m} \ldots V_{n} \ldots V^{(\nu-1)}\right| V_{m+k}+\delta_{m+k-n} w_{m} V_{n} \\
& =\sigma_{m-n} \sum_{j}\left|V_{m} \ldots\left(V_{m+k} P_{j}\right) \ldots V^{(\nu-1)}\right| V_{n} Q_{j}+\left(\sigma_{m-n}+\delta_{m+k-n}\right) w_{m} V_{n}
\end{aligned}
$$

and, summing over $k$ and using Lemma 6.2, we obtain

$$
\left\{w_{m}, V_{n}\right\}=\left(\sigma_{n-m}+\sum_{r=0}^{\nu-1} \phi_{m+r-n}+\sum_{r=1}^{\nu-1} \delta_{m+r-n}\right) w_{m} V_{n}
$$

6.2. Proof of Proposition 1.6. Here the notation $\langle$,$\rangle is used to denote the$ standard scalar product both on $\mathbb{R}^{\nu}$ and on $\mathbb{R}^{\nu-1}$. The following Lemma is used for the proof of the Proposition.

Lemma 6.3. Let $X \in \mathfrak{g}$. Then, in terms of local coordinates $v$ on $\mathbb{R P}^{\nu-1}$, the projective action $X \cdot v$ of $\mathfrak{g}$ on $\mathbb{R P}^{\nu-1}$ is determined by $\langle\alpha, X \cdot v\rangle=\langle(\alpha,-\langle\alpha, v\rangle),(v, 1) X\rangle$ $\forall \alpha \in \mathbb{R}^{\nu-1}$.

Proof. To see this, we projectivise the action of $\exp t X \in G$ on $\mathbb{R}^{\nu}$ and differentiate with respect to $t$. Thus, writing $X=\left(\begin{array}{cc}A & b^{T} \\ c & d\end{array}\right)$ (where $c$ and $b$ are row vectors), 
we get

$$
\begin{aligned}
X \cdot v & =\left.\frac{d}{d t}\right|_{t=0} \frac{1}{1+t d+t v b^{T}}(v+t v A+t c) \\
& =v A+c-d v-\left(v b^{T}\right) v
\end{aligned}
$$

It is now straightforward to check that

$$
\langle\alpha, X \cdot v\rangle=\langle(\alpha,-\langle\alpha, v\rangle),(v, 1) X\rangle .
$$

Proof of Proposition 1.6 For $\alpha$ and $\beta$ some pair of sequences in $\mathbb{R}^{\nu-1}$, let $F, H \in$ $C^{\infty}(\mathcal{W})^{\mathcal{C}}$, with $F(\chi(v, 1))=\langle\alpha, v\rangle$ and $H(\chi(v, 1))=\langle\beta, v\rangle$. Then $d F(v, 1)=$ $(\alpha,-\langle\alpha, v))$ and $d H(v, 1)=(\beta,-\langle\beta, v\rangle)$. Writing, as before, $R=\sum_{i} X_{i} \otimes Y_{i}$ and making use of the Lemma, gives

$$
\begin{aligned}
\{F, H\}(v, 1)= & \sum_{m, n}\left\langle(\alpha,-\langle\alpha, v\rangle)_{m} \otimes(\beta,-\langle\beta, v\rangle)_{n},\left(v_{m}, 1\right) \otimes\left(v_{n}, 1\right)\right. \\
& {\left.\left[R+\sigma_{m-n}(C+I d \otimes I d)+\phi_{m-n}\right]\right\rangle } \\
= & \sum_{m, n} \sum_{i}\left\langle\alpha_{m}, X_{i} \cdot v_{m}\right\rangle\left\langle\beta_{n}, Y_{i} \cdot v_{n}\right\rangle \\
& \quad+\sum_{m, n} \sigma_{m-n}\left(v_{m}^{T}, 1\right)\left(\begin{array}{c}
\beta_{n} \\
-\left\langle\beta_{n}, v_{n}\right\rangle
\end{array}\right)\left(v_{n}^{T}, 1\right)\left(\begin{array}{c}
\alpha_{m} \\
-\left\langle\alpha_{m}, v_{m}\right\rangle
\end{array}\right) \\
& +\sum_{m, n} \phi_{m-n}\left(\left\langle\alpha_{m}, v_{m}\right\rangle-\left\langle\alpha_{m}, v_{m}\right\rangle\right)\left(\left\langle\beta_{n}, v_{n}\right\rangle-\left\langle\beta_{n}, v_{n}\right\rangle\right) \\
= & \sum_{m, n}\left\langle\alpha_{m} \otimes \beta_{n},\left(v_{m} \otimes v_{n}\right) \cdot R\right\rangle+\sum_{m, n} \sigma_{m-n}\left\langle\alpha_{m} \otimes \beta_{n},\left(v_{n}-v_{m}\right) \otimes\left(v_{m}-v_{n}\right)\right\rangle \\
= & \sum_{m, n}\left\langle\alpha_{m} \otimes \beta_{n},\left(v_{m} \otimes v_{n}\right) \cdot R-\sigma_{m-n}\left(v_{m}-v_{n}\right) \otimes\left(v_{m}-v_{n}\right)\right\rangle
\end{aligned}
$$

6.3. Proof of Corollary 2.1. The point of this Corollary is that indeed it follows directly from Proposition [1.6. However it is straightforward to check the claim directly by using the Poisson tensors in their operator forms. The constraint $\rho \equiv 1$ is a second-class constraint for the Poisson structure in (25) (i.e. $\operatorname{det}\left(\left\{\rho_{m}, \rho_{n}\right\}_{\rho \equiv 1}\right) \neq$ 0 ) and we may apply the Dirac formula for a second-class constraint: If in local coordinates $(x, y)$ on a Poisson manifold $\left(x \in \mathbb{R}^{k}, y \in \mathbb{R}^{l}\right.$ say), the constraint is supposed to be to the submanifold given by fixing the $y$-coordinates $y=y_{0}$, then if the Poisson structure has the form

$$
\left(\begin{array}{c}
\dot{x} \\
\dot{y}
\end{array}\right)=\left(\begin{array}{cc}
A & B \\
-B^{*} & C
\end{array}\right) \cdot\left(\begin{array}{c}
\delta_{x} H \\
\delta_{y} H
\end{array}\right)
$$


with $\operatorname{det}\left(\left.C\right|_{y=y_{0}}\right) \neq 0$, the constrained Poisson structure is given in local coordinates $x \in \mathbb{R}^{k}$ by

$$
\dot{x}=\left[A+B C^{-1} B^{*}\right]_{y=y_{0}} \cdot \delta_{x} H
$$

Applying this formula to (25) with $A=D \rho-\rho D^{-1}, B=\mu(D-1) \rho, C=\rho(D-$ $\left.D^{-1}\right) \rho$, yields the constraint of the second Toda Poisson structure to $\rho \equiv 1$ :

$$
\begin{aligned}
\dot{u}=\left(\left.\dot{\mu}\right|_{\rho \equiv 1}\right) & =\left[D-D^{-1}-u(D-1)\left(D-D^{-1}\right)^{-1}\left(1-D^{-1}\right) u\right] \delta_{u} H \\
& =\left[D-D^{-1}-u(D-1)\left(D^{2}-1\right)^{-1}(D-1) u\right] \delta_{u} H \\
& =\left[u\left(\frac{1-D}{1+D}\right) u+D-D^{-1}\right] \delta_{u} H .
\end{aligned}
$$

Generalisation of this proof to the constraint $\rho \equiv \beta$, for some fixed periodic sequence $\beta \in F u n\left(\mathbb{Z} / N \mathbb{Z}, \mathbb{R}^{\times}\right)$, is straightforward.

6.4. Proof of Proposition 2.2. For $H \in C^{\infty}(F u n(\mathbb{Z} / N \mathbb{Z}, \mathbb{R}))$, let $X=\delta_{u} H$, i.e.

$\left.\forall \Delta \in \operatorname{Fun}(\mathbb{Z} / N \mathbb{Z}, \mathbb{R}) \quad \frac{d}{d t}\right|_{t=0} H(u+t \Delta)=\left\langle\Delta, \delta_{u} H\right\rangle=\sum_{n=0}^{N-1} \Delta_{n}\left(\delta_{u} H\right)_{n}=\sum_{n=0}^{N-1} \Delta_{n} X_{n}$.

Then the Hamiltonian vector field $\mathbb{X}_{H}$ is

$$
\dot{u}=\mathbb{X}_{H}(u)=\left(u\left[\frac{1-D}{1+D}\right] u+D-D^{-1}\right) X .
$$

Now consider $H(u)=F\left(u u^{\prime}\right)=F(S) \Rightarrow X=u^{\prime} \delta_{S} F+D^{-1}\left(u \delta_{S} F\right)$. Thus

$$
\begin{aligned}
\dot{S}=\mathbb{X}_{H}(S) & =\dot{u} u^{\prime}+u \dot{u}^{\prime}=\left(u^{\prime}+u D\right) \dot{u} \\
& =\left(u^{\prime}+u D\right)\left(u\left[\frac{1-D}{1+D}\right] u+D-D^{-1}\right)\left(u^{\prime}+D^{-1} u\right) \delta_{S} F .
\end{aligned}
$$

This simplifies to give the result

$$
\begin{aligned}
\dot{S}=(D S+ & S D-D^{-1} S-S D^{-1}+S D^{-1} S-S D S \\
& \left.+S D S^{-1} D S-S D^{-1} S^{-1} D^{-1} S\right) \delta_{S} F
\end{aligned}
$$

as promised.

Again, generalisation to the constraint $\rho \equiv \beta$ is straightforward.

\section{Appendix B: proofs for Section 4}

Before beginning let us recall that the symbol $\stackrel{k}{\vee}$ on a determinant signifies that the $k$ th term is missing. Usually this symbol will be placed at the end, but sometimes 
it will sit at a suitable location in the middle of the determinant. We have

$$
\begin{aligned}
\left\{\alpha_{m}^{(k)}, V_{n}\right\} & =\left\{\left|V_{m} \ldots V_{m+k-1} V_{m+k+1} \ldots V_{m+\nu}\right|, V_{n}\right\} \\
& =\left(\sum_{r=0, r \neq k}^{\nu} \phi_{m-n+r}\right) \alpha_{m}^{(k)} V_{n}+\sigma_{m-n}\left|V_{n} V_{m+1} \ldots V_{m+\nu}\right|^{\vee} V_{m} \\
& +\sigma_{m-n+1}\left|V_{m} V_{n} \ldots V_{m+\nu}\right|^{\vee} V_{m+1}+\cdots+\sigma_{m-n+k-1}\left|V_{m} \ldots V_{n} V_{m+k+1} \ldots V_{m+\nu}\right| V_{m+k-1} \\
& +\sigma_{m-n+k+1}\left|V_{m} \ldots V_{m+k-1} V_{n} \ldots V_{m+\nu}\right| V_{m+k+1}+\ldots+\sigma_{m-n+\nu}\left|V_{m} \ldots V_{n}\right|^{\vee} V_{m+\nu},
\end{aligned}
$$

For $0 \leq r \leq k-1$, let us use Lemma 6.1 and consider the contribution of

$$
\begin{aligned}
& \sigma_{m-n+r}\left|V_{m} \ldots V_{n} \ldots V_{m+\nu}\right|^{\vee} V_{m+r} \\
& \quad=\left(\sigma_{m-n}+\delta_{m-n}+2\left(\delta_{m-n+1} \cdots+\delta_{m-n+r-1}\right)+\delta_{m-n+r}\right)\left|V_{m} \ldots V_{n} \ldots V_{m+\nu}\right|^{\vee} V_{m+r} \\
& =\left(\sigma_{m-n}+\delta_{m-n+r}\right)\left|V_{m} \ldots V_{n} \ldots V_{m+\nu}\right|^{\vee} V_{m+r} .
\end{aligned}
$$

For $k+1 \leq r \leq \nu$, again using Lemma 6.1, consider now the contribution of

$$
\begin{aligned}
& \sigma_{m-n+r}\left|V_{m} \ldots V_{m+k-1} V_{m+k+1} \ldots V_{n} \ldots V_{m+\nu}\right| V_{m+r}= \\
& \left(\sigma_{m-n}+\delta_{m-n}+2\left(\delta_{m-n+1} \ldots+\delta_{m-n+r-1}\right)+\delta_{m-n+r}\right)\left|V_{m} \ldots V_{m+k-1} V_{m+k+1} \ldots V_{n} \ldots V_{m+\nu}\right| V_{m+r} \\
& =\left(\sigma_{m-n}+2 \delta_{m-n+k}+\delta_{m-n+r}\right)\left|V_{m} \ldots V_{m+k-1} V_{m+k+1} \ldots V_{n} \ldots V_{m+\nu}\right| V_{m+r} \\
& =\sigma_{m-n}\left|V_{m} \ldots V_{n} \ldots V_{m+\nu}\right|^{\vee} V_{m+r}+\delta_{m-n+r}\left|V_{m} \ldots V_{m+\nu}\right|^{\vee} V_{n} \\
& \quad+2(-1)^{r-k-1} \delta_{m-n+k}\left|V_{m} \ldots V_{m+\nu}\right|^{\vee v} V_{m+r} \\
& =\sigma_{m-n}\left|V_{m} \ldots V_{n} \ldots V_{m+\nu}\right|^{\vee} V_{m+r} \\
& \quad+\delta_{m-n+r} \alpha_{m}^{(k)} V_{n}-2(-1)^{r-k} \delta_{m-n+k} \alpha_{m}^{(r)} V_{m+r} .
\end{aligned}
$$


Putting all of these together and using Lemma 6.2 in the same way it was used in the proof of Proposition 1.4, we obtain

$$
\begin{aligned}
\left\{\alpha_{m}^{(k)}, V_{n}\right\}= & \left(\sigma_{m-n}+\sum_{r=0}^{\nu} r \neq k\right. \\
\phi_{m-n+r}+\sum_{r=1}^{\nu} r \neq k & \left.\delta_{m-n+r}\right) \alpha_{m}^{(k)} V_{n} \\
& -2(-1)^{\nu-k} \delta_{m-n+k} w_{m}\left(V_{m+\nu}+\sum_{r=1}^{\nu-k-1}(-1)^{\nu-k-r} a_{m}^{(k+r)} V_{m+k+r}\right) \\
= & \left(\sigma_{m-n}+\sum_{r=0}^{\nu} r \neq k\right. \\
& \left.-2(-1)^{\nu-k} \delta_{m-n+k} w_{m} X_{m, n}+\sum_{r=1}^{\nu} r \neq k \delta_{m-n+r}\right) \alpha_{m}^{(k)} V_{n} \\
\text { with } X_{m, n}= & V_{n+\nu-k}+\sum_{r=1}^{\nu-k-1}(-1)^{\nu-k-r} a_{m}^{(k+r)} V_{n+r} .
\end{aligned}
$$

Using the relation (37) this equation may also be written in the alternative form

$$
\begin{aligned}
& \left\{\alpha_{m}^{(k)}, V_{n}\right\}=\left(\sigma_{m-n}+\sum_{r=0}^{\nu}{ }_{r \neq k} \phi_{m-n+r}+\sum_{r=1}^{\nu} r \neq k \delta_{m-n+r}\right) \alpha_{m}^{(k)} V_{n} \\
& +2(-1)^{\nu-k} \delta_{m-n+k} w_{m}\left(\sum_{r=0}^{k}(-1)^{\nu-k+r} a_{m}^{(k-r)} V_{m+k-r}\right) \\
& =\left(\sigma_{m-n}+\sum_{r=0}^{\nu}{ }_{r \neq k} \phi_{m-n+r}+\sum_{r=1}^{\nu}{ }_{r \neq k} \delta_{m-n+r}\right) \alpha_{m}^{(k)} V_{n} \\
& +2 \delta_{m-n+k} w_{m} Y_{m, n} \quad \text { say, } \\
& \text { with } Y_{m, n}=\sum_{r=0}^{k}(-1)^{r} a_{m}^{(k-r)} V_{n-r} .
\end{aligned}
$$

A much simpler computation leads in a similar fashion to

$$
\left\{w_{m}, V_{n}\right\}=\left(\sigma_{m-n}+\sum_{r=0}^{\nu-1} \phi_{m-n+r}+\sum_{r=1}^{\nu-1} \delta_{m-n+r}\right) w_{m} V_{n}
$$

Using (41) we may compute

$$
\left\{w_{m}, w_{n}\right\}=\sum_{l=0}^{\nu-1}\left(\sigma_{m-n-l}+\sum_{r=0}^{\nu-1} \phi_{m-n+r-l}+\sum_{r=1}^{\nu-1} \delta_{m-n+r-l}\right) w_{m} w_{n}
$$

and

$$
\left\{w_{m}, \alpha_{n}^{(k)}\right\}=\sum_{l=0}^{\nu} r \neq k\left(\sigma_{m-n-l}+\sum_{r=0}^{\nu-1} \phi_{m-n+r-l}+\sum_{r=1}^{\nu-1} \delta_{m-n+r-l}\right) w_{m} \alpha_{n}^{(k)}
$$


which implies

$$
\left\{\alpha_{m}^{(k)}, w_{n}\right\}=\sum_{l=0}^{\nu} r \neq k\left(\sigma_{m-n+l}+\sum_{r=0}^{\nu-1} \phi_{m-n-r+l}-\sum_{r=1}^{\nu-1} \delta_{m-n-r+l}\right) \alpha_{m}^{(k)} w_{n} .
$$

Now, using (39) and (40), let us suppose that $k<j$ and compute

$$
\begin{aligned}
& \left\{\alpha_{m}^{(k)}, \alpha_{n}^{(j)}\right\}=\sum_{l=0}^{\nu} l \neq j\left(\sigma_{m-n-l}+\sum_{r=0}^{\nu} r \neq k \phi_{m-n+r-l}+\sum_{r=1}^{\nu}{ }_{r \neq k} \delta_{m-n+r-l}\right) \alpha_{m}^{(k)} \alpha_{n}^{(j)} \\
& -2(-1)^{\nu-k} w_{m}\left(\delta_{m-n+k}\left|X_{m, n} V_{n+1} \stackrel{\stackrel{j}{v} .}{.} V_{n+\nu}\right|\right. \\
& \left.+\delta_{m-n+k-1}\left|V_{n} X_{m, n+1} \stackrel{j}{\vee} V_{n+\nu}\right|+\cdots+\delta_{m-n}\left|V_{n} V_{n+1} \ldots \underset{\substack{\uparrow, n+k \\
k}}{\stackrel{j}{V}} V_{n+\nu}\right|\right) \\
& +2 w_{m}\left(\delta_{m-n-1}\left|V_{n} \ldots Y_{\substack{m, n+k+1 \\
k+1}}^{\stackrel{j}{\vee}} V_{n+\nu}\right|+\cdots+\delta_{m-n+k-\nu}\left|V_{n} \stackrel{\stackrel{j}{V}}{\cdots} Y_{m, n+\nu}\right|\right) .
\end{aligned}
$$

Inspecting this last equation carefully we see that each term is quadratic in the fields $w$ and $\alpha^{(p)}$ for various values of $p$. Moreover, we see that there is no term of the form $\alpha^{(p)} \alpha^{(p)}$. However an almost identical calculation applied to the Poisson bracket $\left\{\alpha_{m}^{(k)}, \alpha_{n}^{(k)}\right\}$ gives the following

Proposition 7.1. When represented in terms of the coordinates a on $\mathcal{W} / G$, for each $k \in\{0,1, \ldots, \nu-1\}$, the quadratic combination $\alpha^{(k)} \alpha^{(k)}$ appears in the entries of the Poisson tensor only in the bracket $\left\{\alpha^{(k)}, \alpha^{(k)}\right\}$ and

$$
\begin{aligned}
& \left\{\alpha_{m}^{(k)}, \alpha_{n}^{(k)}\right\} \\
& =\left(2 \sum_{l=1}^{\nu-k} \delta_{m-n-l}+\sum_{l=0}^{\nu} l \neq k\left[\sigma_{m-n-l}+\sum_{r=0}^{\nu} r \neq k\right.\right. \\
& +\ldots
\end{aligned}
$$

where the dots stand for quadratic terms of the form $\left(\alpha^{(p)} \cdot A_{m, n, p, q} \cdot \alpha^{(q)}\right)$ for $p \neq q$, or $\left(\alpha^{(p)} \cdot B_{m, n, p} \cdot w\right)$, or $\left(w \cdot C_{m, n} \cdot w\right)$.

Proof. The proof is by direct calculation. In fact the appropriate analogue of (45) differs in just one term: the last of the terms containing an $X$ is

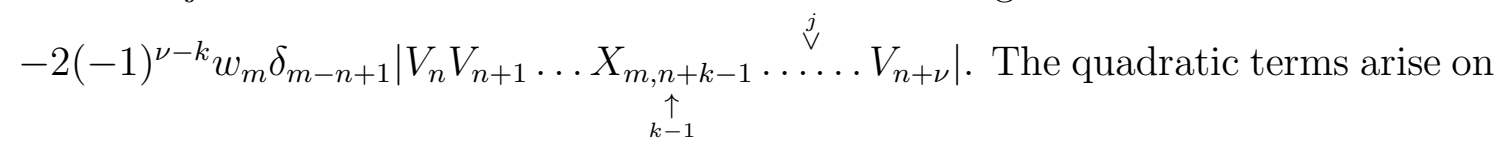
the first line and amongst each of the terms containing a $Y$.

We may now use the formulae (42, 43, 44, 46) and (38) to write down Poisson brackets involving the fields $a^{(k)}$, deciding in advance to discard all terms which are not quadratic in $a^{(k)}$. 
Let us use the operator notation adapted to replacement of the functions $\sigma, \delta, \phi$ by suitable operators, for which the functions are the kernels. For a Poisson bracket relation of the form $\left\{x_{m}, y_{n}\right\}=x_{m} \cdot A_{m-n} \cdot y_{n}$ we denote by $\widehat{\{x, y\}}$ the operator $A$ for which the function $m \mapsto A_{m}$ is the kernel. This notation may be used for the Poisson brackets $\{w, w\}$ and $\left\{w, \alpha^{(k)}\right\}$, but not for $\left\{\alpha^{(k)}, \alpha^{(j)}\right\}$. However we may define $\left\{\widehat{\alpha^{(k)}, \alpha^{(k)}}\right\}$ to be the operator defining the quadratic term. The previous formulae are then written

$$
\begin{aligned}
\widehat{\{w, w\}} & =\sum_{l=0}^{\nu-1} D^{-l} \sigma+\sum_{l=0}^{\nu-1} \sum_{r=0}^{\nu-1} D^{r-l} \phi+\sum_{l=0}^{\nu-1} \sum_{r=1}^{\nu-1} D^{r-l} \\
& =\sum_{l=0}^{\nu-1} D^{-l}(\sigma-1)+\sum_{l=0}^{\nu-1} \sum_{r=0}^{\nu-1} D^{r-l}(\phi+1), \\
\left\{\widehat{w, \alpha^{(k)}}\right\} & =\sum_{l=0}^{\nu} l \neq k \\
\left\{\widehat{\alpha^{(k)}, w}\right\} & =\sum_{l=0}^{\nu} l \neq k \\
\left.\widehat{\alpha^{(k)}, \alpha^{(k)}}\right\} & \left.=\sum_{l=0}^{\nu} l \neq k+1\right)+\sum_{l=0}^{\nu} l \neq k \sum_{r=0}^{\nu-1} D^{r-l}(\phi+1),
\end{aligned}
$$

We have

$\left\{a_{m}^{(k)}, a_{n}^{(k)}\right\}=w_{m}^{-1} w_{n}^{-1}\left(\left\{\alpha_{m}^{(k)}, \alpha_{n}^{(k)}\right\}-a_{m}^{(k)}\left\{w_{m}, \alpha_{n}^{(k)}\right\}-a_{n}^{(k)}\left\{\alpha_{m}^{(k)}, w_{n}\right\}+a_{m}^{(k)} a_{n}^{(k)}\left\{w_{m}, w_{n}\right\}\right)$,

which, after discarding non-quadratic terms, may be rewritten in the form

$$
\left\{\widehat{a^{(k)}, a^{(k)}}\right\}=\left\{\widehat{\alpha^{(k)}, \alpha^{(k)}}\right\}-\left\{\widehat{w, \alpha^{(k)}}\right\}-\left\{\widehat{\alpha^{(k)}, w}\right\}+\widehat{\{w, w\}} .
$$

Now, using (47) we have

$$
\begin{aligned}
\left\{\widehat{a^{(k)}, a^{(k)}}\right\} & =\sum_{l=0}^{\nu} l \neq k D^{-l}(\sigma-1)+\sum_{l=0}^{\nu} l \neq k \sum_{r=0}^{\nu}{ }_{r \neq k} D^{r-l}(\phi+1)+2 \sum_{l=1}^{\nu-k} D^{-l} \\
& +\sum_{l=0}^{\nu-1} D^{-l}(\sigma-1)+\sum_{l=0}^{\nu-1} \sum_{r=0}^{\nu-1} D^{r-l}(\phi+1) \\
& -\sum_{l=0}^{\nu} l \neq k D^{-l}(\sigma-1)-\sum_{l=0}^{\nu} l \neq k \sum_{r=0}^{\nu-1} D^{r-l}(\phi+1) \\
& -\sum_{l=0}^{\nu} l \neq k
\end{aligned}
$$


Distilling the above using the standard formula for geometric series, we obtain

$$
\left\{a^{\widehat{(k)}, a^{(k)}}\right\}=\left(D^{-\nu}-D^{-k}\right)\left[\left(D^{\nu}-D^{k}\right) \phi+\left(D^{\nu}+D^{k}\right)\right] .
$$

In other words

$$
\left\{a^{(k)}, a^{(k)}\right\}=a^{(k)}\left(D^{-\nu}-D^{-k}\right)\left[\left(D^{\nu}-D^{k}\right) \phi+\left(D^{\nu}+D^{k}\right)\right] a^{(k)}+\cdots
$$

where the dots signify terms which are linear in the variables, and the choice

$$
\phi=\frac{D^{k}+D^{\nu}}{D^{k}-D^{\nu}}
$$

forces the Poisson tensor to have linear dependence on $a^{(k)}$.

On the other hand

$$
\left\{a^{(0)}, a^{(k)}\right\}=\frac{1}{w}\left(\left\{w^{\prime}, \alpha^{(k)}\right\}-\left\{w^{\prime}, w\right\} a^{(k)}-a^{(0)}\left\{w, \alpha^{(k)}\right\}+a^{(0)}\{w, w\} a^{(k)}\right) \frac{1}{w}
$$

which may be rewritten in the form

$$
\begin{aligned}
& \left.\left\{\widehat{a^{(0)}, a^{(k)}}\right\}=(D-1)\left\{\widehat{w, \alpha^{(k)}}\right\}-(D-1) \widehat{\{w, w}\right\} \\
& =(D-1)\left(\left\{\widehat{w, \alpha^{(k)}}\right\}-\widehat{\{w, w\}}\right) \text {. }
\end{aligned}
$$

Now, using (47),

$$
\begin{aligned}
\left\{\widehat{w, \alpha^{(k)}}\right\}-\widehat{\{w, w\}} & =\left(D^{-\nu}-D^{-k}\right)(\sigma-1)+\left(D^{-\nu}-D^{-k}\right) \sum_{r=0}^{\nu-1} D^{r}(\phi+1) \\
& =\left(D^{-\nu}-D^{-k}\right)(D-1)^{-1}\left[2+\left(D^{\nu}-1\right)(\phi+1)\right] \\
& =\left(D^{-\nu}-D^{-k}\right)(D-1)^{-1}\left[\left(D^{\nu}-1\right) \phi+\left(D^{\nu}+1\right)\right] .
\end{aligned}
$$

Moreover

$$
\left\{a^{(0)}, a^{(0)}\right\}=\frac{1}{w}\left(\left\{w^{\prime}, w^{\prime}\right\}-\left\{w^{\prime}, w\right\} a^{(0)}-a^{(0)}\left\{w, w^{\prime}\right\}+a^{(0)}\{w, w\} a^{(0)}\right) \frac{1}{w}
$$

so that

$$
\begin{aligned}
\left\{\widehat{a^{(0)}, a^{(0)}}\right\} & =\left(2-D-D^{-1}\right) \widehat{\{w, w\}} \\
& =-D^{-1}(D-1)^{2}\left[D^{1-\nu} \frac{D^{\nu}-1}{D-1} \frac{2}{D-1}+D^{1-\nu}\left(\frac{D^{\nu}-1}{D-1}\right)^{2}(\phi+1)\right] \\
& =-\left(1-D^{-\nu}\right)\left[2+\left(D^{\nu}-1\right)(\phi+1)\right] \\
& =\left(D^{-\nu}-1\right)\left[\left(D^{\nu}-1\right) \phi+\left(D^{\nu}+1\right)\right] .
\end{aligned}
$$

Hence the choice

$$
\phi=\frac{1+D^{\nu}}{1-D^{\nu}}
$$

forces $a^{(0)}$ to be a Casimir. 


\section{REFERENCES}

[A] Adler, M., On a trace functional for formal pseudodifferential operators and the symplectic structure of the Koteweg-de Vries type equations, Inv. Math. 50 (1979), 219-248.

[B] Babelon, O. Exchange formula and lattice deformation of the Virasoro algebra, Phys. Lett. B 238 (1990), no. 2-4, 234-238.

[BFP] Balog, J., Feher, L., Palla, L. On the Chiral WZNW phase space, exchange r-matrices and Poisson-Lie groupoids pp. 1-19 in: Integrable Systems: From Classical to Quantum, eds. J. Harnad et al, AMS, 2000, arXiv:hep-th/9912173

[C] Carlet, G. The extended bigraded Toda Hierarchy J. Phys. A 39 (2006) 9411-9435

[CDZ] Carlet, G., Dubrovin, B., Zhang, Y., The extended Toda hierarchy Mosc. Math. J. 4 (2004) 313-332

[D] Dubrovin, B., Private communication.

[DZ] Dubrovin, B., Zhang, Y., Virasoro symmetries of the extended Toda hierarchy Commun. Math. Phys. 250 (2004) 161-193

[DS] Drinfeld, V.G., Sokolov, V.V. Lie algebras and equations of Korteweg-de Vries type, Itogi Nauk. Tekh. Sov. Probl. Mat. 24 (1984) 81-180; J. Sov. Math. Dokl. 30 (1985) 1975-2036.

[F] Faddeev, L.D. Quantum symmetry in Conformal Field Theory by Hamiltonian methods in "New Symmetry Principles in Quantum Field Theory", (Plenum Press, New York) NATO ASI Series Physics 295 (1992) 159-174.

[FT] Faddeev, L.D., Takhtajan, L.A. Liouville model on the lattice, Lect. Notes in Phys. 246 (1986), 166-179.

[FRS] Frenkel, E., Reshetikhin, N., Semenov-Tian-Shansky, M.A. Drinfeld-Sokolov reduction for difference operators and deformations of $W$-algebras. I: The case of Virasoro algebra, Commun. Math. Phys. 192 (1998) 605-629.

[GD] Gelfand, I.M., Dickey, L.A., Family of Hamiltonian structures connected with integrable non-linear equations, Collected papers of I.M. Gelfand, vol. 1, Springer-Verlag (1987), 625-646.

[L] Lu, J.-H., Momentum mappings and reduction of Poisson actions in "Symplectic Geometry, Groupoids and Integrable Systems" (Berkeley, Calif., 1989), Math. Sci. Res. Inst. Publ., vol. 20, Springer, New York, 1991, 209-226

[LP] Li, L.-C., Parmentier, S. Nonlinear Poisson structures and r-matrices, Comm. Math. Phys. 125 (1989) 545-563

[MS] Marshall, I., Semenov-Tian-Shansky, M.A. Poisson groups and differential Galois theory of Schrödinger equation on the circle, Commun. Math. Phys. 284 (2008) 537-552

[OR] Oevel, W., Ragnisco, O. R-matrices and higher Poisson brackets for integrable systems, Phys. A 161 (1989) 181-220

[OST] Ovsienko, V., Schwartz, R., Tabachnikov, S. The Pentagram map: a discrete integrable system arXiv:0810.5605 [math.DS]

[S1] Semenov-Tian-Shansky, M.A. Dressing action transformations and Poisson-Lie group actions, Publ. RIMS. 21 (1985), 1237-1260.

[SS] Semenov-Tian-Shansky, M.A., Sevostyanov, A.V. Drinfeld-Sokolov reduction for difference operators and deformations of $W$-algebras. II: The general semisimple case, Commun. Math. Phys. 192 (1998) 631-647.

[VS] Veselov, A.P., Shabat, A.B. Dressing chain and spectral theory of the Schrödinger operator, Funktsional. Anal. i Prilozhen. 27 (1993) 1-21; Functional Analysis and its Applications 27 (1993), 81-96.

[V] Volkov, A.Yu. Miura transformation on a lattice, Theor. Math. Phys. 74 (1988), 96-99.

Mathematics Department, University of Loughborough, UK

E-mail address: ian.marshall@unige.ch 